\title{
Acceso por vía cubital como alternativa al acceso radial para diagnóstico e intervenciones coronarias: serie de 29 pacientes
}

\author{
Manuel Méndez ${ }^{1-2}$, Osvaldo Pérez ${ }^{1-2}$, Leoncio León ${ }^{1}$, Francisco Ramírez ${ }^{1}$, Mery Mestas $^{1}$, Karla Zuanic ${ }^{1}$, \\ Alejandro Martínez
}

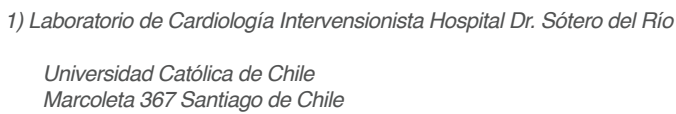

\section{Resumen:}

Objetivo: Evaluar la seguridad y resultados de los procedimientos coronarios por vía cubital.

Pacientes y Método: Se evaluó en forma retrospectiva todos los pacientes sometidos a procedimientos diagnósticos y terapéuticos por vía cubital en el laboratorio de hemodinamica del hospital Dr. Sotero del Río entre el 29 de Enero del 2009 y el 31 de Agosto del 2010. Todos los pacientes fueron sometido a prueba de Allen birideccional antes del estudio y en todos se intentó el acceso radial como primera alternativa. Se registraron características tanto clínicas como del procedimiento. Todos los pacientes fueron seguidos clínicamente al alta y en forma telefónica.

Resultados : De un total del 1937 procedimientos coronarios 29 fueron por vía cubital $(1,49 \%) .12$ pacientes fueron sometidos a angioplastía, 6 de ellas primaria, 17, $2 \%$ de los pacientes resultaron diabéticos, 51,7\% hipertensos e igual porcentaje de mujeres.

Todos los procedimientos fueron exitosos. No hubo complicaciones isquemicas ni hemorrágicas al momento del alta. No se registraron complicaciones clínicas en el seguimiento alejado a 6,5 meses promedio.

Conclusión : El acceso por vía cubital con vaina 6 french es una alternativa segura y eficaz tanto para diagnostico angiográfico como para procedimientos terapéuticos, independiente del contexto clínico del paciente. 


\section{The ulnar approach as an alternative to the radial access for coronary angiography and coronary interventions. A pilot study on 29 patients.}

Aim: to evaluate the safety and feasibility of the ulnar percutaneous approach for cardiac catheterization as an alternative to the radial access in every day practice.

Patients and Methods: a retrospective evaluation was performed in 29 patients undergoing a coronary angiography or intervention using the ulnar approach after a failed radial attempt, between January 2009 and August 2010. A 6 French sheath was used in all patients. Clinical and procedure related events were evaluated at discharge, at 30 days and in the month of August 2010.

Results: the 29 patients constituted $1.5 \%$ of the total

\section{Introducción:}

El acceso por punción radial para diagnóstico y terapia coronaria se ha transformado en la vía de elección en muchos centros dado la baja tasa de complicaciones vasculares, la comodidad y confort para el paciente y la posibilidad de deambulación precoz que se traducen en menor estadía hospitalaria. Sin embargo, la variabilidad anatómica y de tamaño, así como la inadecuada irrigación colateral, no siempre hacen posible el acceso por esta arteria ${ }^{1-6}$.

Desde que en el 2001 Terashima $^{7}$ reportara el uso de la arteria cubital o ulnar para diagnóstico y terapia coronaria, esta arteria se ha transformado en una alternativa en pacientes seleccionados aún cuando las características anatómicas, principalmente la cercanía con el nervio cubital y su profundidad la hacen potencialmente propensa a complicaciones diferentes a las de la arteria radial.

Dos estudios aleatorios han comparado este acceso con la punción por vía radial demostrando la efectividad y seguridad de la punción ulnar en cerca de 400 pacientes con tasas de éxito y oclusión arterial del todo comparables $^{8-9}$.

El acceso radial es la vía por defecto en nuestro centro luego de un período de entrenamiento que incluyó a más de 200 procedimientos por cada operador. En algunos casos, tras fracasar el acceso radial hemos utilizado el acceso cubital como alternativa natural de esta técnica.

\section{Objetivo}

Evaluar la seguridad y resultados del acceso cubital (ulnar) como alternativa a la punción radial en una serie de 29 pacientes consecutivos realizados number of procedures performed in the corresponding time interval. 12 of the 29 were PTCAs, 6 of them in the setting of acute myocardial infarction. Diabetes was present in $17.2 \%$, hypertension in $51.7 \%$ and $51.7 \%$ were women. The procedures ended without events in every patient. No vascular or hemorrhagic complications were observed during follow up.

Conclusion: the ulnar approach can be safely used as an alternative to radial access for coronary angiography and intervention

Key words: ulnar vascular access, coronary artery interventions.

en nuestro centro.

\section{Pacientes y método}

Se evaluó en forma retrospectiva todos los pacientes sometidos a procedimientos diagnósticos y terapéuticos por vía cubital en el laboratorio de cardiología intervencionista del hospital Dr. Sótero del Río entre Enero del 2009 y Agosto del 2010. Todos los pacientes fueron sometido a prueba de Allen bidireccional antes del estudio, todos los pacientes tenían arteria cubital palpable y en todos se intentó sin éxito la punción radial como primera alternativa. Nunca se puncionó la cubital del mismo lado si es que se había instalado un introductor en la radial correspondiente o el pulso radial había desaparecido, lo que se consideró como criterio de exclusión.

Se registraron las características clínicas y del procedimiento. Todos los pacientes fueron seguidos clínicamente al alta y en forma telefónica a los 30 días y al año. En aquellos pacientes en que el tiempo de seguimiento fue de entre 30 días y un año se les realizó una encuesta telefónica durante el mes de agosto del 2010.

Seis pacientes fueron sometidos a doppler de las arterias de la mano correspondiente.

\section{Punción cubital y procedimiento}

La punción se realizó con el brazo extendido al costado de cuerpo luego de la aplicación subcutánea de 2 ó 3 cc de lidocaína al $5 \%$ dentro de los $2 \mathrm{~cm}$ proximales al hueso pisiforme sobre un segmento de la arteria palpable usando el set de punción e introductor radial hidrofílico Terumo (Terumo Corporation). La punción se rea- 
lizó con técnica transfixiante, tal como se recomienda para este set. Todos los introductores utilizados fueron 6 french $(\mathrm{F})$. La canulación arterial selectiva para diagnóstico se realizó con catéteres Judkins izquierdo(JL) y derecho (JR) $5 \mathrm{~F}$. Las angioplastías se realizaron con catéteres 6 F. Todos los pacientes recibieron $5000 \mathrm{U}$ de heparina I.V. al inicio del procedimiento y se completó la dosis hasta alcanzar $100 \mathrm{U} / \mathrm{kg}$ en caso de angioplastía. Todos los introductores fueron retirados inmediatamente terminado el procedimiento aplicando compresión con la banda neumática TR Bandtm (Terumo Corporation) por dos a tres horas, posterior a lo cual se dejó un apósito semi compresivo por 4 a 6 horas. El alta de los pacientes se programó según el diagnóstico de ingreso.

\section{Resultados}

De un total de 1937 pacientes sometidos a estudio coronario en el período antes detallado, 29 fueron por acceso cubital $(1,49 \%)$. En todos los casos en que se intentó la punción ulnar, ésta fue exitosa. Las características clínicas de los pacientes se observan en la tabla 1.

En los 29 pacientes se realizó angiografía diagnóstica. Todas las inyecciones fueron selectivas y se lograron con catéteres JL 3,5 para mujeres y 4 para hombres, en el caso del árbol coronario izquierdo, y JR 4 para la arteria coronaria derecha. Salvo en un caso en que la arteria coronaria derecha se originaba en el seno coronario izquierdo, no se utilizaron más catéteres que los descritos (canulación diagnóstica y terapéutica con Amplatz izquierdo y derecho respectivamente).

Se realizaron 12 angioplastías, 6 de ellas primarias. Ocho angioplastías se realizaron sobre territorio dependiente del tronco coronario común izquierdo utilizando en todas catéter guía Launcher EBU 3,5 (catéter de primera elección), 4 de ellas sobre la arteria descendente anterior.

Cuatro angioplastías se realizaron sobre la arteria coronaria derecha utilizando en tres de ellas catéter guía Judkins derecho curva 4 y en una ocasión un catéter Launcher Amplatz derecho curva 2 (caso descrito en párrafo anterior). Todos los procedimientos fueron exitosos. En 21 pacientes el acceso fue vía cubital derecha, en los 8 restantes se accedió desde la izquierda, ya sea porque el procedimiento se inició por ese lado o por la calidad del pulso cubital.

Ningún paciente presentó espasmo clínico relevante de la arteria cubital.

No se registró ninguna complicación del sitio de punción al alta ni en el seguimiento promedio de 6,5 meses.
(1-14 meses, mediana de 8 meses).

Seis pacientes (3 de ellos angioplastía) fueron sometidos a doppler de la arteria correspondiente entre uno y 9 meses después del procedimiento; ninguno presentó oclusión de la arteria.

\begin{tabular}{|c|c|c|}
\hline Tabla 1 & $\mathbf{N}$ & $\%$ \\
\hline $\begin{array}{l}\text { Mayores } 75 \text { a os } \\
\text { Mediana edad (a os) } \\
\text { Promedio edad (a os) } \\
\text { Diabetes Mellitus } 2 \\
\text { Hipertensi n arterial } \\
\text { Tabaquismo } \\
\text { S ndrome coronario agudo } \\
\text { Mujeres } \\
\text { Angioplast a } \\
\text { Angioplast a primaria }\end{array}$ & $\begin{array}{l}3 \\
60 \\
57,8 \\
5 \\
15 \\
8 \\
15 \\
15 \\
12 \\
6\end{array}$ & $\begin{array}{c}17,2 \\
51,7 \\
27 \\
51,7 \\
51,7 \\
41,3 \\
20,5\end{array}$ \\
\hline
\end{tabular}

\section{Discusión}

El acceso por vía radial para diagnóstico y terapia coronaria ha demostrado ser más seguro que el femoral, principalmente, por la disminución de las complicaciones en el sitio de punción ${ }^{10}$. Nuestro centro accede en algo más del $96 \%$ de los casos por esta vía.

Si bien el número de procedimientos por vía cubital dio cuenta de algo menos del 1,5\% del total de procedimientos realizados, en nuestra serie no se presentaron complicaciones del sitio de punción, así como tampoco evidencias clínicas de trauma del ulnar, lo que está en concordancia con lo descrito en series electivas y estudios aleatorizados recientes ${ }^{8-9-11}$, confirmando que el acceso ulnar es tan seguro como el radial. Sin embargo, ninguna de estas series incluyó angioplastías primarias. Doce de los pacientes fueron sometidos a angioplastía utilizándose los catéteres de alto flujo habituales según la elección del operador no presentándose ninguna dificultad en la manipulación de éstos. Seis de estas angioplastías fueron durante el transcurso de infarto con supradesnivel del segmento ST reforzando la idea de la disponibilidad de este acceso en situaciones de la práctica clínica diaria.

La arteria cubital suele ser de mayor diámetro que la arteria radial y con menor número de receptores alfa lo que explicaría la facilidad para puncionarla y la ausencia de espasmo clínicamente relevante como ya ha sido reportado con anterioridad ${ }^{11}$.

La curva de aprendizaje del cateterismo por vía radial es 
más larga que por vía femoral.

La experiencia no publicada de nuestro grupo confirman que la tasa de fracasos es inversa al número de procedimientos y a la complejidad de éstos, experiencia que es del todo aplicable al acceso por vía ulnar.

Un buen tema a debatir es la necesidad de realizar este acceso en los casos en que la radial no es accesible. Está demostrado que las complicaciones, sobre todo hemorrágicas, son menores por vía radial y que los resultados son comparables al acceso cubital ${ }^{8-9}$. A nuestro juicio, parece del todo necesario extremar lo que se ha denominado erróneamente como "acceso desde el carpo" 12 .

La tasa de oclusión radial varía en torno al 5\% pero el proceso de cicatrización así como el daño no oclusivo sobre la arteria hacen que otra razón discutible para usar la vía cubital es la de preservar la radial ya sea para ser utilizada como injerto libre durante cirugía de revascularización miocárdica o en pacientes sometidos a diálisis, en la fabricación de fístulas arterio venosas.

\section{Limitaciones}

Desde luego se trata de una serie retrospectiva que si bien incluye pacientes no seleccionados, en todos ellos la arteria cubital era palpable no registrándose el número de pacientes que, frente a una punción radial frustra, no fue posible palpar la cubital teniendo que cambiar el acceso. Otra limitación se refiere a la ausencia de registro de tiempos del procedimiento y de radioscopia o dosis total de radiación, así como la falta de imágenes que certifiquen la permeabilidad de la arteria en un porcentaje mayor de pacientes.

\section{Conclusión}

El acceso por vía cubital con vaina 6 french es una alternativa segura y eficaz tanto para diagnóstico angiográfico, como para procedimientos terapéuticos, independiente del contexto clínico del paciente.

\section{Referencias:}

1. KIEMENEIJ F, LAARMAN GJ. Percutaneous transradial artery approach for coronary Palmaz-Schatz stent implantation. Am Heart J 1994; 128: 167-174.

2. CRUDEN NL, TEH CH, STARKEY IR, NEWBY DE. Reduced vascular complications and length of stay with transradial rescue angioplasty for acute myocardial infarction. Cathet Cardiovasc Intervent 2007; 70: 670-675.

3. CHASE AJ, FRETZ EB, WARBURTON WP, KLINKE WP, CARERE RG, PI D, et al. Association of the arterial access site at angioplasty with transfusion and mortality: the MORTAL study (Mortality benefit Of Reduced Transfusion after percutaneous coronary intervention via the Arm or Leg). Heart 2008; 94: 1019-1025.

4. FEIT F, VOELTZMD,ATTUBATO MJ,LINCOFFAM, CHEW DP, BITTL JA, et al. Predictors and impact of major hemorrhage on mortality following percutaneous coronary intervention from the REPLACE-2 Trial. Am J Cardiol 2007; 100: 1364-1369.

5. RAO SV, OU FS, WANG TY, ROE MT, BRINDIS R, RUMSFELD JS, et al. Trends in the prevalence and outcomes of radial and femoral approaches to percutaneous coronary intervention: a report from the National Cardiovascular Data Registry. JACC Cardiovasc Interv 2008; 1: 379-386.

6. NAGAI S, ABE S, SATO T, HOZAWA K, YUKI K, HANASHIMA K, et al. Ultrasonic assessment of vascular complica- tions in coronary angiography and angioplasty after transradial approach. Am J Cardiol 1999; 83: 180-186. Cardiovasc Interv 2004; 61: 56-59.

7. TERASHIMA M, MEGURO T, TAKEDA H, ENDOH N, ITO Y, MITSUOKAM, et al. Percutaneous ulnar artery approach for coronary angiography: a preliminary report in nine patients. CatheterCardiovasc Interv 2001; 53: 410-414.

8. LI YZ, ZHOU YJ, ZHAO YX, GUO YH, LIU YY, SHI $\mathrm{DM}$, et al. Safety jand efficacy of transulnar approach for

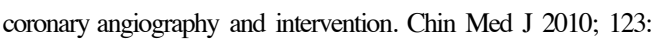
1774-1779

9. APTECAR E, PERNES JM, CHABANE-CHAOUCH M, BUSSY N, CATARINO G, SHAHMIR A, et al. Transulnar versus transradial artery approach for coronaryangioplasty: the PCVI-CUBA study. Catheter Cardiovasc Interv. 2006; 67: 711-20

10. AGOSTONI P, BIONDI-ZOCCAI GG, DE BENEDICTIS ML, RIGATTIERI S, TURRI M, ANSELMI M, et al. Radial versus femoral approach for percutaneous coronary diagnostic and interventional pro- cedures; systematic overview and meta-analysis of randomized trials. J Am Coll Cardiol 2004; 44: 349-56.

11. KNEBEL AV, CARDOSO CO, CORREA RODRIGUES LH, SARMENTO-LEITE RE, DE QUADROS AS, MASCIA GOTTSCHALL CA, et al. Safety and Feasibilityof transulnar cardiac Catheterization. Tex Heart Inst J 2008; 35: 268-72.

12. TORSTEN SCHWALN. Transcarpal catheterization. Dtscl Arztebl int 2009; 106: 685-91 